\title{
Pharmacoepigenetics and pharmacoepigenomics
}

"Advances in epigenetics and epigenomics are impacting pharmacology, leading to the development of new specialities, pharmacoepigenetics [and pharmacoepigenomics]"

The recent developments in genetics and genomics, such as the Human Genome Project and the International HapMap Project, have led to advances in pharmacogenetics and pharmacogenomics [1]. The developments in genetics and genomics have also led to major advances in two other fields: epigenetics and epigenomics. Epigenetics refers to the study of heritable changes in gene expression without any changes in DNA sequence. Epigenetics involves three interacting molecular mechanisms: DNA methylation, modification of histones in chromatin and RNA-mediated regulation of gene expression [2]. Epigenomics refers to the study of epigenetics on a genome-wide basis. Interest in epigenomics has led to the Human Epigenome Project, the systematic study of epigenetic changes across the human genome in health and disease [3]. Epigenetic patterns are known to be reversible and vary with age as well as varying from tissue to tissue, since an individual has multiple epigenomes [4]. The importance of epigenetics in clinical medicine is being increasingly appreciated, both in the pathogenesis of single-gene disorders and common diseases [5,6]. Among the common diseases, except for cancer, little is known at present regarding the role of epigenetics in the pathogenesis of these diseases [6].

Advances in epigenetics and epigenomics are impacting on pharmacology, leading to the development of new specialities, pharmacoepigenetics [7], the study of the epigenetic basis for variation in drug response and pharmacoepigenomics [4]. Pharmacoepigenomics involves the study of the role of epigenomics on intrapersonal and interpersonal variations in response of individuals to drugs; on the effects of drugs on geneexpression profiles; in the mechanisms of action of drugs and adverse drug reactions; and in the discovery of new drug targets. It has been predicted that in the future, pharmacoepigenomics will have an ever-increasing role in pharmacology and clinical medicine [4]. Researchers now have access to a burgeoning collection of tools for unraveling the epigenome [8]. This bodes well for the future of pharmacoepigenetics and pharmacoepigenomics.

A major component of pharmacoepigenetics and pharmacoepigenomics is epigenetic therapy, the use of drugs to treat and prevent epigenetic defects associated with disease [2]. To date, epigenetic drugs that have been studied in the greatest detail are inhibitors of DNA methyltransferase and histone deacetylase and these drugs have mainly been studied for their use in the management of cancer [9]. Drugs from both these drug classes have started receiving approval from the US FDA for treatment of patients. A major problem with epigenetic drugs is nonspecificity of action, resulting in effects on genes that are not desired targets, leading to adverse effects like carcinogenicity [9]. Although to date, most research on epigenetic drugs has been on developing inhibitors of DNA methyltransferase and histone deacetylase, there are also other potential targets for these drugs like histone acetyltransferase and histone methyltransferase [10]. Andrew Feinberg has suggested that instead of using epigenetic drugs for the treatment of disease, one can target biochemical pathways, that have been disturbed, epigenetically in disease using more conventional drugs [5].

In addition to extensive ongoing work on DNA methyltransferase and histone deacetylase inhibitors, at present there is also extensive work in trying to develop drugs associated with the other aspect of epigenetics: RNA-mediated regulation of gene expression. This phenomenon refers to the regulation of gene expression by small noncoding RNAs of which there are several types like small interfering RNAs (siRNAs) and microRNAs (miRNAs) [11]. The precursors of miRNAs and siRNAs are longer double stranded RNAs that are processed to small RNAs by dedicated sets of enzymes and other proteins. Although in some conditions small RNAs can activate geneexpression, they are mainly involved in silencing gene-expression [11]. Small RNAs are known to regulate the expression of more than $30 \%$ of

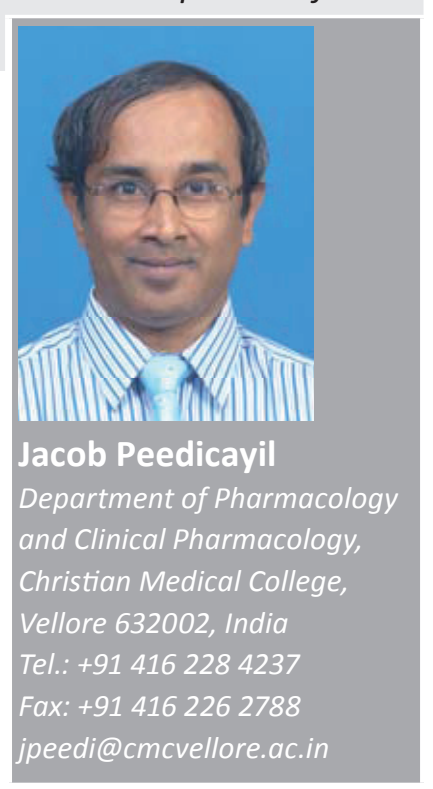

future inedict $\mathrm{fSg}$ 
protein-coding genes by blocking mRNA translation, an action that may be accompanied by mRNA degradation [12,13]. Aberrant miRNA expression is known to contribute to the pathogenesis of diseases such as cancers and cardiac disease and can hence serve as novel targets for therapy [12]. For example, antisense oligonucleotides complementary to specific miRNAs (antimiRNA ASOs, also called antagomirs) may be useful in therapy [12].

\section{"A major component of pharmacoepigenetics and pharmacoepigenomics is epigenetic therapy"}

Small interfering RNAs can potentially be used in a new form of therapy, RNA interference (RNAi), which can be triggered by two different pathways. One pathway involves a RNA-based approach where synthetic effector siRNA duplexes are delivered by various carriers to target cells [13]. The other pathway involves a DNA-based approach where the siRNA effectors are produced by intracellular processing of longer RNA hairpin transcripts. This approach is mainly based on nuclear synthesis of another type of small RNA called short-hairpin RNAs, which are transported to the cytoplasm via the miRNA export pathway and then processed into siRNAs [13,14]. Several pharmaceutical companies across the world are presently attempting to make RNAi therapeutics a reality for use in clinical conditions [15]. At present, difficulties with RNAi include off-target effects as well as appropriately delivering the siRNA to the desired cell or tissue [13].

It has been predicted that the better understanding of the interplay between DNA methylation, histone modification and RNA-mediated regulation of gene expression which is expected to result from the Human Epigenome Project, will lead to a better understanding of human disease and a new range of molecular targets for epigenetic drugs [3]. Although epigenetic therapy to date has been most studied for its use in cancer there are many other diseases for which it may be useful such as mental [2], cardiovascular [5] and inflammatory [16] diseases. Epigenetic mechanisms in addition to being involved in the pharmacological effects of drugs, are also known to be involved in the toxic effects of drugs and chemicals [17]. Just as the application of genetics and genomics to toxicology led to the fields of toxicogenetics [18] and toxicogenomics [19], the application of epigenetics and epigenomics to toxicology may, in the future, lead to new disciplines called toxicoepigenetics and toxicoepigenomics.

\section{Financial \& competing interests disclosure}

The author has no relevant affiliations or financial involvement with any organization or entity with a financial interest in or financial conflict with the subject matter or materials discussed in the manuscript. This includes employment, consultancies, honoraria, stock ownership or options, expert testimony, grants or patents received or pending, or royalties.

No writing assistance was utilized in the production of this manuscript.

\section{Bibliography}

1 Shurin SB, Nabel EG:

Pharmacogenomics - ready for prime time?

N. Engl. J. Med. 358(10), 1061-1063 (2008).

2 Peedicayil J: Epigenetic therapy - a new development in pharmacology. Indian J. Med. Res. 123(1), 17-24 (2006).

3 The American Association for Cancer Research Human Epigenome Task Force and the European Union, Network of Excellence, Scientific Advisory Board: Moving AHEAD with an international human epigenome project. Nature 454, 711-715 (2008).

4 Szyf M: Toward a discipline of pharmacoepigenomics. Curr. Pharmacogenomics 2(4), 357-377 (2004).

5 Feinberg AP: Epigenetics at the epicenter of modern medicine. JAMA 299 (11), 1345-1350 (2008).

6 Feinberg AP: Phenotypic plasticity and the epigenetics of human disease. Nature 447 , 433-440 (2007).
7 Ingelman-Sundberg M, Sim SC, Gomez A, Rodriguez-Antona C: Influence of cytochrome P450 polymorphisms on drug therapies: pharmacogenetic, pharmacoepigenetic and clinical aspects. Pharmacol. Ther. 116(3), 496-526 (2007).

8 Bonetta L: Epigenomics: detailed analysis. Nature 454, 795-800 (2008).

9 Gal-Yam EN, Saito Y, Egger G, Jones PA: Cancer epigenetics: modifications, screening, and therapy. Annu. Rev. Med. 59, 267-280 (2008).

10 Kristeleit R, Stimson L, Workman P, Aherne W: Histone modification enzymes: novel targets for cancer drugs. Expert Opin. Emerg. Drugs 9(1), 135-154 (2004).

11 Grosshans H, Filipowicz W: Molecular biology: the expanding world of small RNAs. Nature 451, 414-416 (2008).

12 Zhang B, Farwell MA: microRNAs: a new emerging class of players for disease diagnostics and gene therapy. J. Cell. Mol. Med. 12 (1), 3-21 (2008).
13 Kim DH, Rossi JJ: RNAi mechanisms and applications. Biotechniques 44(5), 613-616 (2008).

14 Hannon GJ, Rossi JJ: Unlocking the potential of the human genome with RNA interference. Nature 431, 371-378 (2004).

15 Haussecker D: The business of RNAi therapeutics. Hum. Gene Ther. 19(5), 451-462 (2008).

16 Adcock IM: HDAC inhibitors as antiinflammatory agents. Br. J. Pharmacol. 150 (7), 829-831 (2007).

17 Watson RE, Goodman JI: Epigenetics and DNA methylation come of age in toxicology. Toxicol. Sci. 67(1), 11-16 (2002).

18 Orphanides G, Kimber I: Toxicogenetics: applications and opportunities. Toxicol. Sci. 75(1), 1-6 (2003).

19 Hamadeh HK, Amin RP, Paules RS, Afshari CA: An overview of toxicogenomics. Curr. Issues. Mol. Biol. 4(2), 45-56 (2002). 